\title{
El suicidio en un destino turístico: Cozumel
}

DOI: 10.22403/UQROOMX/TYP05/25

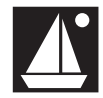

Resumen

Karina Amador Soriano*

El presente artículo es un proyecto de investigación que tiene el propósito de establecer si existe una relación entre las causas que originan el suicidio y la localidad en que ocurre (este caso Cozumel, en el Caribe mexicano). El suicidio se ha estudiado desde el ámbito científico de la Psiquiatría, la Psicología, la Medicina y actualmente con mayor amplitud desde la Sociología. El suicidio es un fenómeno importante visto desde la lupa sociológica en una región donde el turismo es la principal actividad económica, lo que a su vez genera conductas sociales particulares de la entidad.

Palabras | Conductas desviadas, depresión, estrés, frustración, marginaCLAVE ción social, suicidio. 


\section{Introducción}

Cuando se habla del suicidio se establecen dos posibles causas extrasociales que lo motivan: las disposiciones orgánico-psíquicas y la naturaleza del medio físico (Durkheim, 1998), las primeras se refieren a la necesidad de terminar con una vida insatisfecha, donde aparecen los trastornos mentales, siendo la depresión el síntoma principal. En la segunda se encuentran el clima, la temperatura, etc., dada la forma en que actúan sobre el organismo.

Los mensajes subliminales en los medios de comunicación son un ejemplo de la presión social que ejerce el entorno en personas predispuestas al suicidio: "Si no vas a Liverpool no eres importante","Si no te vistes con la marca Calvin Klein seguramente no existes", “Si tienes más de 30 años ya te quedaste solo (a)”. El suicida reconoce poco su existencia y no se da cuenta de que él o ella están en condiciones de reconstruir constantemente sus aspiraciones y crear una vida en donde "él / ella" albergue sentido y no sólo simple felicidad.

El incremento del suicidio ha provocado que distintas disciplinas se den a la tarea de explicar sus causas y consecuencias, por ejemplo: la Historia nos remonta a ritos de las primeras culturas prehispánicas; la Filosofía nos introduce en el estudio del ser y la vida y la muerte; la Teología juzga que el suicidio es un pecado; la Psicología trata de dar una explicación integral, es decir desde el entorno personal, familiar y social del suicida; la Sociología es aún más amplia, su investigación se vuelca hacia el individuo y las relaciones sociales que establece con su medio, es decir la influencia que generan los sistemas sociales para que se llegue al suicidio. Es esta última disciplina la que sirve de base para el presente análisis.

\section{Planteamiento del problema}

Vivimos en un mundo globalizado que se caracteriza por la innovación humana y el progreso tecnológico, además del desplazamiento de personas (mano de obra) y la transferencia de conocimientos (tecnología) a través de las fronteras internacionales (FMI, 2000). La globalización abarca además aspectos culturales, políticos y ambientales que imponen modas, estilos de vida y formas de pensamiento, entre otros; una consecuencia es el estrés, el cual tiene efectos sobre la salud mental: frustración, ansiedad y depresión. Asimismo, hay otras formas 
en que el estrés puede manifestarse: alcoholismo, farmacodependencia $y$, en casos extremos, el suicidio (Sebastián García, 2002).

La Organización Mundial de la Salud (OMS) reporta que el suicidio constituye un problema de salud pública; en 2004 cerca de la mitad de las muertes violentas fueron resultado del suicidio, lo que equivale a casi un millón de víctimas al año:

"Las estimaciones realizadas indican que en 2020 las víctimas podrían ascender a 1.5 millones [...] cada suicidio supone la devastación emocional, social y económica de numerosos familiares y amigos", ha declarado la doctora Catherine Le Galès-Camus, subdirectora general de Enfermedades No Transmisibles y Salud Mental de la OMs, "el suicidio es un trágico problema de salud en todo el mundo, se producen más muertes por suicidio que por la suma de homicidios y guerras." [OMs, Centro de prensa, 2004].

Las tasas más altas de suicidio se dan en Europa del Este, y las más bajas en América Latina; se calcula que por cada muerte atribuible a esa causa se producen entre diez y 20 intentos, que se manifiestan en lesiones, hospitalizaciones y traumas emocionales y mentales, pero no se dispone de datos fiables sobre su verdadero alcance. Las tasas tienden a aumentar con la edad, pero recientemente se ha registrado en todo el mundo un incremento alarmante de los comportamientos suicidas entre los jóvenes de 15 a 25 años, matándose más hombres que mujeres, aunque la mayoría de los intentos de quitarse la vida son más frecuentes entre las personas de sexo femenino (Coespo et al., 2007).

En México, en las últimas décadas, las estadísticas oficiales y los centros médicos (como Cruz Roja y Hospital General) evidencian el incremento del número de individuos que se privan de la vida en el país. El Instituto Nacional de Estadística, Geografía e Informática (INEGI) reporta que la cuarta causa de mortalidad es el suicidio. Como veremos en las siguientes tablas, la primera causa de muerte es por accidentes, seguido de homicidios y tumores malignos, y en cuarto lugar el suicidio (cuadro I).

Al contrastar las causas de mortalidad entre hombres y mujeres se concluye que los primeros se suicidan más que las segundas, aunque, como ya se mencionó, éstas lo intentan con mayor frecuencia (cuadro 2).

Un estudio realizado por el INEGI en 2004, con base en los datos de todas las agencias del Ministerio Público de México, con respecto a suicidios por 
El suicidio en un destino turístico: Cozumel

Cuadro I. Porcentaje de defunciones de 15 a 29 años, sus CAUSAS de 2003 a 2005

\begin{tabular}{lccc}
\hline Causas & $\begin{array}{c}2003 \\
\%\end{array}$ & $\begin{array}{c}2004 \\
\%\end{array}$ & $\begin{array}{c}2005 \\
\%\end{array}$ \\
\hline Accidentes & 32.1 & 31.9 & 32.1 \\
Agresiones (homicidios) & 12.9 & 12.0 & 12.4 \\
Tumores malignos & 8.0 & 8.5 & 8.2 \\
$\begin{array}{l}\text { Lesiones autoinflingidas } \\
\text { intencionalmente (suicidio) }\end{array}$ & 6.4 & 6.4 & 6.5 \\
$\begin{array}{l}\text { Enfermedades por virus de } \\
\text { inmunodeficiencia humana }\end{array}$ & & & \\
\hline
\end{tabular}

Fuente: INEGI, 2005.

Cuadro 2. Causas de MORTALIDAD EN HOMBreS Y MUJeRES DE 15 a 29 años

\begin{tabular}{ll}
\hline \multicolumn{1}{c}{ Hombres } & \multicolumn{1}{c}{ Mujeres } \\
\hline Accidentes & Accidentes \\
Agresiones (homicidios) & Tumores malignos \\
$\begin{array}{l}\text { Lesiones autoinflingidas } \\
\text { intencionalmente (suicidios) }\end{array}$ & Embarazo, parto y puerperio \\
Tumores malignos & Agresiones (homicidios) \\
Enfermedad por virus de la & Lesiones autoinflingidas \\
inmunodeficiencia humana & intencionalmente (suicidios) \\
\hline
\end{tabular}

Fuente: INEGI, 2005

zona, reportó que ocho de cada diez de estas muertes ocurren en las zonas urbanas (Jalisco y Distrito Federal) y dos de ellas en las zonas rurales (Hidalgo registró $82 \%$ de suicidas). Las dos causas principales, según este organismo, son: disgustos familiares y relaciones amorosas (Martínez, 2005).

El Estado de Quintana Roo ocupa el sexto lugar nacional en suicidios (Noticaribe, 2007); las causas principales son problemas con la pareja, consumo de drogas y alcohol, y violencia intrafamiliar (Martoccia, 2006). En los últimos 
datos obtenidos para esta investigación se encontró que en 2006 la cifra en la entidad alcanzó 105 suicidios, y las edades en que las personas se privan de la vida es entre 19 y 35 años, Cancún y Chetumal tienen los números más elevados en la estadística estatal; una cifra aún más reciente habla de que hasta mayo de 2007 se habían reportado 127 suicidios (Diario de Quintana Roo, 30 de mayo de 2007).

Las autoridades consideraron que para finales del 2007 en Cancún se habrían matado I 20 personas. En su reporte de mayo de 2007, el INEGI anunció que, de los tres estados que integran la Península de Yucatán, Quintana Roo ocupa el primer lugar en suicidios.

De lo anterior se obtiene una evidencia importante para la investigación: por un lado, los suicidios se cometen en dos polos: en la ciudad de Chetumal, rodeada de comunidades rurales e indígenas, donde el índice turístico es menor, y en Cancún, donde el turismo ocupa el primer lugar en la economía que impulsa a la entidad.

La isla de Cozumel no es ajena al tema del suicidio, en el 2005 hubo seis decesos, en el 2006 aumentaron a ocho,y, hasta julio de 2007, I I personas se habían quitado la vida.'

La situación socioeconómica y demográfica de la isla presenta un crecimiento poblacional de $9 \%$ en los últimos cinco años, la industria hotelera genera empleos y atrae inmigración de los estados vecinos -principalmente de Chiapas y Campeche- (González Canto, 2005). Una de las consecuencias del crecimiento es el hacinamiento, que genera una falta de privacidad y da como resultado conductas no deseables: estrés familiar, violencia intrafamiliar, entre otras. Según datos del año 2006 del Observatorio Urbano de la Riviera Maya, sólo $2 \%$ de las familias de Cozumel que están integradas por cuatro miembros viven en espacios adecuados (tres cuartos), y $13 \%$ del mismo número de integrantes se tiene que acomodar en dos habitaciones (Frausto, 2006: I0I).

El comportamiento suicida está determinado por un gran número de causas, el objetivo de la presente investigación es establecer las principales. Lo que es evidente es que se está convirtiendo en un problema serio para la entidad.

\footnotetext{
'Información obtenida del Ministerio Público del Fuero Común, Cozumel, Quintana Roo, julio de 2007.
} 
El suicidio en un destino

turístico: Cozumel

\section{Justificación}

El estado de Quintana Roo se localiza en la porción oriental de la Península de Yucatán, colinda al norte con Yucatán y el Golfo de México; al este con el mar Caribe; al sur con la bahía de Chetumal, Belice y la República de Guatemala, y al oeste con Campeche.Al estado lo integran ocho municipios: Felipe CarriIlo Puerto, Isla Mujeres, Othón P. Blanco, Benito Juárez, José María Morelos, Lázaro Cárdenas, Solidaridad y Cozumel. Su capital es Chetumal y el clima es cálido húmedo con lluvias abundantes en verano.

Quintana Roo experimenta una marcada división económica; por un lado el glamour de sus zonas turísticas, con hoteles de lujo (Cancún, Riviera Maya, Cozumel, etc.) y, por el otro, áreas rural-urbanas como Chetumal, Felipe Carillo Puerto, José María Morelos, etc. Las zonas turísticas registran una elevada concentración urbana y altas tasas de inmigración de personas procedentes, en la mayoría de los casos, de entidades vecinas, debido a la significativa derrama económica que hay en el estado (González Canto, 2005).

Cozumel, isla del Caribe, ubicada a $19 \mathrm{~km}$ al oeste de la costa de Quintana Roo, es la más grande de México, con una extensión de $647.33 \mathrm{~km}^{2}$, una temperatura media anual de $26^{\circ} \mathrm{C}$, y un clima cálido húmedo con abundantes lluvias en verano. Está cubierta por una vegetación selvática, rodeada por costas rocosas interrumpidas por playas de color marfil, lagunas y manglares, en cuyas profundidades puede observarse a simple vista un importante conjunto de barreras de coral pertenecientes al arrecife maya, el segundo sistema de arrecifes del mundo, sobre el que se han instituido reservas naturales de gran valor ecológico como el Parque Marino Nacional Arrecifes de Cozumel y el Parque Natural de Chankanaab, zonas de gran biodiversidad marina que han llamado la atención de investigadores y científicos como Jacques Cousteau y en las que pueden practicarse actividades como el buceo y el ecoturismo. ${ }^{2}$

El turismo en Cozumel deja una cuantiosa derrama económica a la entidad y un nulo índice de desempleo, pues sus restaurantes, agencias de viajes, la producción y venta de artesanías, las tiendas de buceo y una variada gama de empresas dedicadas al ramo turístico son altamente generadoras de fuentes de empleo.

La isla tiene 78193 habitantes (INEGI, 2005), de los cuales $77.4 \%$ son hombres, y $29.4 \%$ mujeres. La población económicamente activa (PEA) suma

${ }^{2}$ Información tomada de: Consejo de Promoción Turística, Sectur 2007, disponible en: www.visitmexico.com/wb/Visitmexico/Visi_Cozumel (consultado el I4 de mayo de 2007). 
7I\%. Lo anterior nos deja claro que el desempleo no es factor de trascendencia, sin embargo una tasa de desempleo reducida no asegura que los habitantes cubran sus necesidades básicas, e incluso puede ser motivo para omitir responsabilidades. Otros datos, obtenidos del Observatorio Urbano de la Riviera Maya (Frausto, 2006: 17 y 86), indican que la isla presenta un crecimiento poblacional anual de $3.8 \%$, lo cual genera una demanda adicional de servicios básicos, infraestructura, vivienda accesible, etc. En este último rubro es preciso mencionar que sólo $55.7 \%$ de la población tiene vivienda propia, mientras que $27 \%$ renta, $9.6 \%$ paga la casa que habita, y $6.8 \%$ ocupa una vivienda prestada -es decir que viven en casas de padres, abuelos, tíos u otros-. El ingreso mensual de las familias cozumeleñas en promedio es de \$4 925 pesos (en un solo ingreso, es decir del hombre o la mujer), cuando los dos tienen empleos remunerados es del doble, lo cual no es común, por lo menos en las colonias que presentan rezagos educativos o laborales, así lo reporta el INEGI como resultado del conteo que se realizó en el 2005.

Con los datos anteriores se vislumbran problemas específicos que son las variables de la investigación: la forma de vida de los habitantes -familiar, económica, laboral-, y el hacinamiento y la migración, entre los más importantes.

La actividad turística constituye un marco donde entran en contacto bagajes culturales y socioeconómicos muy diferentes, los impactos socioculturales son resultado de dichas relaciones sociales (OMT, 1996), los cuales se expresan en la convivencia y adaptación constantes a la dinámica de un destino turístico, en este caso Cozumel.

Es oportuno mencionar que el marco teórico en el que se basa la investigación es el enfoque psicosocial de Emile Durkheim, pionero en las investigaciones sobre el suicidio, quien además enmarcó el comportamiento del individuo en las estructuras sociales, o hechos sociales como él los llama, explicando las causas extrasociales que mueven a un individuo hacia el suicidio. El método sociológico de Durkheim permite a la presente investigación ordenar las ideas y los aspectos dinámicos que se vayan encontrando.

Por todo lo expuesto, se pretende encontrar resultados en dos planos: el primero, el psicosocial, en particular de las causas y consecuencias del efecto suicida; el segundo es el que se refiere a Cozumel como destino turístico y los alcances del suicidio en este lugar. De esta manera se podrían promover proyectos sociales en pro de la prevención y atención por parte de las autoridades municipales correspondientes. 
El suicidio en un destino

turístico: Cozumel

\section{Metodología}

El tipo de investigación es mixta o multimodal, llamada así por ser un enfoque que integra tanto elementos de corte cuantitativo como cualitativo. En el primero se aplicarán encuestas a fin de que surjan datos estadísticos útiles para establecer con exactitud patrones de comportamiento en la población con respecto a la opinión del tema "suicidio". En el segundo enfoque (el cualitativo) se realizarán entrevistas a familiares de personas que han muerto quitándose la vida. El objetivo es tratar de reconstruir la realidad que nos permita comprender este complejo fenómeno. Se establecerá el contacto por medio de personas que conozcan a dichas familias, además de búsquedas en los registros del Ministerio Público del Fuero Común. También se hablará con quienes hayan tenido la intención de suicidarse (suicidas fallidos), para ello se recurrirá a organizaciones no gubernamentales (ONG) en Cozumel, por ejemplo:Alcohólicos Anónimos, Centro de Integración Juvenil, Alcance Victoria, con la finalidad de conocer los motivos que los orillaron a tomar tal decisión. Otras entrevistas de gran trascendencia son las que se realizarán a expertos que trabajan en algunos de estos centros, a fin de contar con información especializada.

El alcance del estudio es de corte exploratorio-descriptivo, por ser uno de los primeros trabajos que se realiza en la entidad. Las fuentes son primarias y secundarias; las primarias (encuestas) consistirán en la opinión de la población tomada como muestra estratificada, personas de 20 a 40 años, que es la edad en la que oscilan los suicidas de la entidad, según las cifras del Observatorio de Violencia Social y de Género en Benito Juárez, Q. R. (Coespo, et al., 2007), sin importar su estado civil o escolaridad. Se seleccionarán con base en la fórmula propuesta por Laura Fischer (2002) sobre las poblaciones finitas.

Las instituciones de Cozumel a las cuales se acudirá para la recopilación de información son: el Ministerio Público de Fuero Común, el Centro de Integración Juvenil, la Cruz Roja Mexicana y el Hospital General de Salubridad. La instituciones de asistencia social (Ayuda en Movimiento, 2005) donde se realizarán entrevistas son: AL-ANON,Aprendiendo a Vivir;Alcance Victoria,A. C.; Alcohólicos Anónimos Cozumel, 3er. Distrito; Centro de Integración Juvenil,A. C.; Fundación Aviomar,A. C.; y Neuróticos Anónimos.

El contenido de la encuesta versará sobre las causas que originan el suicidio, y la opinión con respecto a la relación del suicidio con Cozumel como destino turístico. La guía de la entrevista recopilará datos acerca de las causas 
psicosociales que deja el suicidio en la entidad, el índice que va en aumento y el papel de las instituciones que deben abordar la problemática.

Posteriormente se realizará una base de datos que arrojará graficas que serán analizadas; en cuanto a las entrevistas, se examinarán las respuestas con base en conceptos y contenidos, para hacer la interpretación y análisis de los datos, tomando en cuenta el marco teórico.

\section{Conclusión}

Según datos de la Dirección de Desarrollo Urbano (Ayuntamiento de Cozumel, 2006) la isla presenta un crecimiento poblacional de $9 \%$ en los últimos cinco años. Sin embargo, hay que mencionar que la capacidad poblacional de Cozumel era de 50 a 60000 habitantes, en la actualidad se rebasa la cifra llegando a cerca de 80000 pobladores, las consecuencias se pueden notar en todos los ámbitos: servicios de salud, de infraestructura, de educación. El desarrollo urbano crece a pasos agigantados dejando tras de sí problemas sociales que hasta hace poco tiempo no eran tan notorios: delincuencia, alcoholismo, violencia intrafamiliar, etcétera.

Cozumel es un lugar turístico, donde la industria hotelera o las constructoras generan empleos en sus distintos sectores, lo que produce que personas de estados vecinos migren hacia ella, con la esperanza de mejorar su situación laboral y económica; pero tanto hombres como mujeres llegan a la isla dejando en sus lugares de origen a sus familias, lo cual les provoca tristeza, que en ocasiones suele ser un pretexto para ingerir bebidas alcohólicas, lo que a su vez conlleva otras consecuencias sociales.

Ya mencionamos que según datos del Observatorio Urbano de la Riviera Maya en Cozumel, $2 \%$ de las familias de cuatro miembros vive en espacios adecuados, pero $13 \%$ vive en casas de uno a dos cuartos por el mismo número de personas o hasta dos más. El hacinamiento resta privacidad a los miembros de la familia, y ello redunda en irritación en el trato, que se traduce en violencia intrafamiliar, uno de los factores que ha sido motivo para cometer el acto suicida.

El suicidio es un problema de salud pública, y, por lo tanto, los gobiernos federal, estatal y municipales deben establecer iniciativas sociales para contrarrestarlo. Las investigaciones realizadas en la entidad pueden albergar un proyecto común. Las instituciones escolares pudieran implantar programas de 
prevención o atención. El suicidio aumenta cada día, si bien en Cozumel aún no es un fenómeno "relevante", es importante realizar una investigación que busque prevenirlo.

\section{FUENTES CONSULTADAS}

Ayuntamiento de Cozumel (2006), Índices de población en Cozumel México: Dirección de Desarrollo Urbano,Ayuntamiento de Cozumel.

Canché, C. Ruby E., Odalys Ancona V. y Víctor Hugo Venegas (2005). Ayuda en Movimiento. México: Fundación Aviomar,A.C.

Consejo Estatal de Población (Coespo) "Quintana Roo; Redes: Investigación para el Desarrollo y Observatorio deViolencia Social y de Género en Benito Juárez, Quintana Roo (2007)" [en línea]. Situaciones de suicidio. Tomo II. Disponible en: http://www.sinviolencia.org.mx/sv/enclosures/ EstSuicidio-Tomo2.pdf [2007, 29 de junio].

Diario de Quintana Roo (2007). “Cancún y Chetumal repunte estadística estatal de suicidio” [en línea]. Diario de Quintana Roo, 30 de mayo de 2007. Disponible en: $w w w . d q r . c o m . m x / i n d e x . p h p$ ?option=com_frontpage\& Itemid= I \&month=5\&year=2007 [2007, $\left.\right|^{\circ}$ de junio].

Durkheim, Emile (1998). El suicidio. México: Grupo Editorial Tomo.

Fischer, L. y J. Espejo (2002). Casos de marketing ( $2^{\mathrm{a}}$ ed.). México: Prentice Hall.

Fondo Monetario Internacional (FMI) (2000). La globalización ¿amenaza u oportunidad? [en línea]. Disponible en:http://www.imf.org/external/np/exr/ ib/2000/esl/04I 200s.htm [2007, 7 de junio].

Frausto, Oscar (2006). Sistema de Indicadores de Violencia Social y de Género, Cozumel, 2006 / Hábitat II [en línea]. Observatorio Urbano de la Riviera Maya, Cozumel. Disponible en: <www.cozumel.uqroo.mx/oulrm/cozumel/documentos/PDF/SI-Violencia-Social-Genero-Cozumel-2006. pdf>

González Canto, Félix Arturo (2005). Plan Estatal de Desarrollo 2005-20 I I [en línea]. Gobierno del Estado de Quintana Roo. Disponible en: www. quintanaroo.gob.mx/qroo/index/php [2007, 30 de julio].

Instituto Nacional de Estadística Geografía e Informática (INEGI) (2005). II Conteo de Población y Vivienda 2005 [en línea]. Estadísticas de Mortalidad. 
Disponible en; <http://www.inegi.org.mx/inegi/default. aspx?s=est\&c= I | 094>, [2007, 15 de junio].

2002-2006 “Defunciones, causas" [en línea], Disponible en:www.inegi. gob.mx/est/contenidos/espanol/rutinas/ [2007, I6 de mayo].

Martínez, Ivonne (2005). “Crecen los suicidios en México” [en línea]. Notimex. Disponible en: <www.terra.com.mx/articulo.aspx?articuloid=| 77827 \&paginaid $=1>$ [2007, 17 de junio].

Martoccia, Hugo (2006). "Aumenta 50\% en cuatro años la tasa de suicidios en Quintana Roo” [en línea]. La Jornada, I I de febrero de 2006. Disponible en: www.jornada.unam.mx/2006/02/I I/037nlest.php [2007, I6 de mayo].

Noticaribe (2007). "Arman plan para detener suicidios" [en línea]. Noticaribe, 23 de mayo de 2007. Disponible en: <www.noticaribe.com.mx/chetumal/2007/05/arman_plan_para_detener_suicidios.html>.

OMs, Centro de prensa (2004). "El suicido un problema de salud pública y sin embargo es prevenible" [en línea]. Organización Mundial de la Salud, Ginebra, 8 de septiembre de 2004. Disponible en: $<w w w . w h o . i n t / m e-$ diacentre/news/releases/2004/pr6I/es/> [2007, 5 de junio].

OMT (1996). Introducción al turismo. Madrid: Organización Mundial del Turismo Ponteriro Vitale, Sergio (2003). Metodología en el turismo. México:Trillas.

Sebastián García, Olga (2002). "Los riesgos psicosociales y su prevención: mobbing, estrés y otros problemas" [en línea]. ponencia presentada en la Jornada Técnica de Actualización de los Riesgos Psicosociales y su Prevención: Mobbing, Estrés y Otros Problemas, Madrid, 10 de diciembre de 2002. Disponible en:www.mtas.es/insht/research/POSSebastian.htm,[2007, 8 de junio]. 


\section{Primer Congreso Internacional \\ de Ciencias Sociales}

\section{en el Sureste Mexicano}

\section{3, 24 y 25 de octubre de 2008 Universidad del Caribe Cancún, Quintana Roo}

\section{Mesas de trabajo}

- Antropología, cultura e identidad

- Violencia estructural, género y familia

- Economía y Turismo

- Desarrollo y gobierno locales

- Desarrollo humano e investigación educativa

- Desarrollo urbano y medioambiente

Mayores informes

Mtra. Graciela Vázquez Flores

Departamento de Desarrollo Humano Universidad del Caribe

gvazquez@ucaribe.edu.mx
Mtro. Antonio Higuera Bonfil

Departamento de Ciencias Sociales Universidad de Quintana Roo anthigue@uqroo.mx
Dra. Paloma Escalante G.

Posgrado en Antropología Social Escuela Nacional de Antropología e Historia egpaloma@yahoo.com
